\section{Cap senior salaries to boost junior posts}

In my view, US universities should be refocusing resources on the next generation of academic researchers. For example, they might consider a cap for salaries derived from university funds and repurpose these resources for support of junior faculty positions.

High salaries for administrative staff and senior faculty members contribute to the dearth of available university resources, sequestering tuition, philanthropic and grant-overhead monies. Administrators, as well as many senior scientists with minimal research, teaching or clinical responsibilities, can be paid extraordinarily high annual salaries of up to four times the US National Institutes of Health cap of US\$185,100.

If a $\$ 350,000$ cap on annual salaries for professors of all levels were imposed just within the University of California system (http://ucpay.globl.org), this would provide $\$ 175,309,313$ (figures from 2014 statistics). Such a sum could fund more than 800 assistant-professor positions at about $\$ 200,000$ per year.

Reallocating university resources in this way could create a more hopeful and sustainable future for the next generation of researchers and educators. Improving support for early-career researchers, who are fighting for their livelihoods, would also relieve one of the drivers for insufficient scientific rigour.

Christopher J. Evans University of California, Los Angeles, USA. cevans@g.ucla.edu

\section{China's energy rush harming ecosystem}

Northwest China's 'Energy

Golden Triangle' is being targeted as part of a national strategy to concentrate large-scale energy industries in the region. Given its scarce rainfall and vegetation, it is ecologically fragile and susceptible to atmospheric pollution - a point that seems to have been overlooked in the scramble for long-term energy security.

A team at Lanzhou University has observed signs of water and soil contamination, and damage to the sparse local ecosystem by toxic substances from energy and chemical industries in the area (unpublished results). Sulfur dioxide pollution is increasing there and elsewhere in northwest China (Y. Shen et al. Environ. Sci. Technol. Lett. 3, 275-279; 2016), whereas it has declined across the rest of China in the past decade.

We appeal to the government to undertake in-depth scientific assessments of the environmental consequences of energy activity and development in the region.

Jianmin Ma Lanzhou University, Lanzhou, China.

Jianzhong Xu Northwest Institute of Eco-Environment and Resources, Chinese Academy of Sciences, Lanzhou, China. jianminma@lzu.edu.cn

\section{A European position on genome editing}

The ethics committee of the French national biomedical research agency (INSERM) has put forward recommendations to foster responsible use of genome-editing technologies (see go.nature.com/2fozqad), such as CRISPR-Cas9. This follows a December 2015 meeting of the US National Academy of Sciences, the US National Academy of Medicine, the Chinese Academy of Sciences and the UK Royal Society to produce guidelines for gene editing in humans.

Our recommendations include setting up a European committee of experts from different disciplines to assess the scope, efficacy and safety of CRISPR-Cas9, and reviewing the ban on all genetic modifications to the germline (Article 13 of the Oviedo
Convention). The United States, China and some countries in Europe have not endorsed this ban (T. Ishii Trends Mol. Med. 21, 473-481; 2015).

Other proposals include assessing the freedom of research and medical ethics at European institutions in the context of national and international initiatives, and launching a monitoring group of relevant stakeholders to promote open debate on the societal aspects of these technologies (see, for example, Nature 522, 20-24; 2015).

These measures should assist translation into European and international legislation within the next couple of years.

François Hirsch, Yves Lévy INSERM, Paris, France.

Hervé Chneiweiss INSERM, Sorbonne Universities, UPMC, CNRS, IBPS, NPS, Paris, France. herve.chneiweiss@inserm.fr

\section{India needs agency for energy data}

India has no central body for maintaining and disseminating energy data, let alone analysing it. We propose the creation of a national Energy Information Agency to replace the current patchwork of systems. This would collect, standardize and analyse data across energy domains and make it publicly available.

A lack of robust data sets has hampered India's energy research, undermining policy and regulatory compliance. Data from different bodies, some statutory, are often reported as gross averages and lack timeliness; they may be incomplete and vary in accuracy, and depend on different methodologies and assumptions.

A central Energy Information Agency should coordinate with existing data holders, such as the Central Electricity Authority and the Petroleum Planning and Analysis Cell, and with state and private industry. This would encourage the development of new business models and other innovations (see, for example, E. Funkhouser et al. Energy Res. Soc. Sci. 10, 90-101; 2015).

The agency would help India to expand its energy infrastructure and to improve the availability, affordability and reliability of its energy supply. Frameworks for measuring, reporting and verification (MRV) are also key to honouring India's commitments under the Paris climate deal. Rahul Tongia Brookings India, New Delhi, India.

Varun Rai University of Texas at Austin, USA.

Gireesh Shrimali Stanford University, California, USA. rtongia@brookingsindia.org

\section{A few words can make a big impact}

As postdocs applying for faculty positions, we observe that brief opinion pieces in high-impact journals can be a significant factor in landing a job - even though they cannot compare with painstaking research contributions to more-specialized journals. These short comments signal a crucial ability to step back from one's own narrow field and use scientific knowledge to provide thoughtful perspectives on issues of broad relevance.

Although meticulous work is necessary to push the boundaries of the known a little farther, paying attention to the big picture is especially important for young scientists. Having spent years becoming increasingly specialized, once in a while we need to rise above such technical issues as solving stochastic differential equations or measuring coleopteran wings.

Making it into a high-impact journal, even in an unrefereed section, is an indicator that a specialist can still generate some sensible consideration of value to the global community.

Dario Del Giudice, Andrew

B. Davies Carnegie Institution for Science, Stanford, California, USA. ddelgiudice@carnegiescience.edu 